$91 \mid 2020$

Ivresse patrimoniale ? - Décors d'Intolérance Germaine Dulac - Alice au pays des merveilles - Éric Rondepierre

\title{
Aude Joseph, avec la collaboration de Roland Cosandey, Neuchâtel. Un canton en images. Filmographie Tome 2 (1950-1970)
}

Stéphanie E. Louis

\section{OpenEdition \\ Journals}

Édition électronique

URL : https://journals.openedition.org/1895/8078

DOI : $10.4000 / 1895.8078$

ISSN : $1960-6176$

Éditeur

Association française de recherche sur l'histoire du cinéma (AFRHC)

Édition imprimée

Date de publication : 1 juin 2020

Pagination : 186-188

ISBN : 978-2-37029-091-5

ISSN : 0769-0959

Référence électronique

Stéphanie E. Louis, « Aude Joseph, avec la collaboration de Roland Cosandey, Neuchâtel. Un canton en images. Filmographie Tome 2 (1950-1970) », 1895. Mille huit cent quatre-vingt-quinze [En ligne], 91 | 2020, mis en ligne le 10 mai 2021, consulté le 01 juin 2021. URL : http://journals.openedition.org/1895/8078 ; DOI : https://doi.org/10.4000/1895.8078

Ce document a été généré automatiquement le 1 juin 2021

(c) AFRHC 


\section{Aude Joseph, avec la collaboration de Roland Cosandey, Neuchâtel. Un canton en images. Filmographie Tome 2 (1950-1970)}

Stéphanie E. Louis

\section{RÉFÉRENCE}

Aude Joseph, avec la collaboration de Roland Cosandey, Neuchâtel. Un canton en images. Filmographie Tome 2 (1950-1970), Neuchâtel, Institut neuchâtelois, Éditions Alphil, 2019, $490 \mathrm{p}$.

1 Le premier tome de la filmographie du canton de Neuchâtel est paru en 2008 aux éditions Gilles Attinger et traitait la période 1900-1949. Il recensait 199 films, dont 59 n'avaient été documentés que par des sources secondaires. Le présent volume se concentre sur les deux décennies suivantes et évoque près de 300 titres réalisés entre 1949 et 1969. Il est l'œuvre d'Aude Joseph, responsable des archives audiovisuelles pour le canton de Neuchâtel, en collaboration avec Roland Cosandey, auteurs qui avaient déjà été associés à Christine Rodeschini pour le premier tome.

2 Ce deuxième tome recense des films sur ou réalisés à Neuchâtel, conservés dans différentes collections suisses. L'ouvrage compte 272 notices. Si deux notices collectives se rattachent à des ensembles de spots publicitaires, les autres sont établies par titre. Elles comportent a minima une description technique (titre, année de réalisation; format, couleurs/noir et blanc, métrage, durée; instances de production; lieu de conservation et lieu de consultation) et une description détaillée des contenus sonores et visuels. Les notices les plus élaborées intègrent aussi un commentaire, donnant des compléments d'information sur le réalisateur ou les circonstances de production, voire des références bibliographiques. Des photogrammes en couleur illustrent les notices 
des films conservés au DAV-département audiovisuel de la ville de La Chaux-de-Fonds, initiateur de ce projet. Ce repérage visuel permet, d'une manière à la fois utile et agréable à l'œil, d'articuler la démarche filmographique généralisante à l'inventaire de la collection locale. Les fiches se déclinent chronologiquement puis par ordre alphabétique de titre.

En début de volume, une note explicative vient éclairer la méthodologie appliquée et la typologie des informations indiquées. Une préface par la directrice de la bibliothèque de la ville de La Chaux-de-Fonds, Sylvie Béguelin, résume le contexte institutionnel dans lequel s'inscrit cette parution. Puis, un texte de Roland Cosandey, intitulé «1950-1970 - une filmographie neuchâteloise, ou les motifs dans le tapis » introduit le volume par une remarquable synthèse des enjeux et perspectives de cette initiative.

Cosandey pose la différence entre "patrimoine cinématographique» local - en l'occurrence la conservation de tous les films produits à Neuchâtel - et "patrimoine audiovisuel cantonal» (soit: les documents portant un regard particulier sur «le territoire et ses représentations $»)$, précisant leurs implications en matière de préservation mais aussi les limites d'une application trop hermétique de cette distinction (p. 18). Les grandes lignes du corpus sont présentées et mises en contexte. La carrière de cinéastes du cru comme Henry Brandt, Jean-Pierre Guéra ou André Paratte est rapprochée des dynamiques cinématographiques neuchâteloises (clubs, salles et critique), dont participent aussi les pratiques amateurs. Attirant l'attention sur la diversité des formats coexistant dans le corpus (du 8 au $35 \mathrm{~mm}$ ), Cosandey invite à s'intéresser à la localisation des séances, aux publics auxquels étaient adressés ces films et à leur circulation. S'il s'agit de "considérer les films répertoriés ici comme une source pertinente pour l'étude de la représentation du canton de Neuchâtel [...] une attention préalable à ce qui les différencie » est nécessaire (p.29). L'éventail de ces distinctions va de la nature des films eux-mêmes au lieu de leur conservation, aspects qui sont généralement corrélés. Ainsi le Ciné-Journal Suisse est-il conservé à un échelon national par les Archives fédérales et la Cinémathèque suisse, contrairement aux bandes amateures. Autre élément contextuel nécessaire à l'appréhension du corpus recensé, bien qu'il n'y soit pas inclus : la production télévisuelle en plein essor sur la période considérée, et dont le volume aurait rendu impossible la publication papier (1755 entrées). On s'accorde à dire que «ce volumineux corpus bouleverse considérablement le paysage hétérogène dessiné par la filmographie proprement cinématographique [et] ouvre également, par son exemplarité, des pistes nouvelles à la recherche» (p. 31).

5 En fin d'ouvrage, on trouve de bien utiles index : des thèmes, des personnes, des noms propres (personnes morales, lieux, etc.), et enfin alphabétique des films. La table des matières les reprend quant à elle par ordre chronologique, reflétant l'organisation de l'ouvrage.

6 Ce livre s'accompagne d'un coffret comportant deux DVD coédités par la Bibliothèque de la Ville de La Chaux-de-Fonds et la Cinémathèque suisse. On y trouve 29 films sur le canton de Neuchâtel, groupés en cinq thèmes. "Autour de la montre " évoque l'industrie horlogère avec des films publicitaires ou apparentés à cette tradition, comme Solidarité horlogère (Victor Borel, 1951) commandé par la Chambre suisse de l'horlogerie. «Les Amateurs - de la chronique locale à l'expérimentation" met en lumière la production éclectique des clubs de cinéastes amateurs, qui connurent leur essor à cette période. Des films comme Sarabande et variations (1964) soulignent leur 
inventivité. Son réalisateur, Gilbert Vuillème, est un autodidacte qui se lança dans la réalisation du premier film d'animation dû à un Neuchâtelois et son œuvre fut présentée dans plusieurs festivals internationaux. «Terroirs » montre les paysages et artisanats, tout en évoquant certaines structures ou personnalités engagées dans la valorisation du territoire local (PAC Film, de Jean-Pierre Guéra et René Junod ; André Paratte ou Jean Borel). "L'air du temps" nous plonge dans certains des débats sociétaux qui traversèrent le canton à l'époque: la question du nucléaire et la résistance anti-atomique, mais aussi la traque des bouilleurs de cru clandestins par la Régie fédérale des alcools... «Neuchâtel dans le concert des cantons: Ciné-journal suisse 1950-1960 » présente un florilège de sujets d'actualités filmées, qui documentent des rassemblements de jeunes, des manifestations culturelles ou sportives, les inaugurations de locaux ou d'équipements collectifs, des commémorations. Deux autres curiosités cinématographiques s'ajoutent à ces ensembles thématiques. «En guise d'introduction ", une publicité pour Suchard commandée au cinéaste allemand Hans Fischerkoeser vers 1950. "Pour conclure", comme un flash-back sur ce projet filmographique de longue haleine, un fragment de Fête fédérale de lutte et de jeux alpestres, film réalisé autour de 1908. Si le film était évoqué dans le premier tome, ce fragment n'a été retrouvé qu'en 2011 à la Stiftung Deutsche Kinemathek de Berlin. Déposé à présent à la Cinémathèque suisse, il constitue «le plus ancien film neuchâtelois conservé». Un livret d'une cinquantaine de pages, bien illustré et commenté, accompagne les films. Notons, pour ceux qui souhaiteraient se replonger totalement dans cette filmographie neuchâteloise qu'un premier DVD a été publié sur la période 1910-1950, également dans la collection «Le Cinéma des régions » de la Cinémathèque suisse.

7 L'ensemble apparaît donc comme un exemple réussi de coopération entre des organismes de conservation autour de patrimoines filmiques régionaux, qui documentent la vitalité économique, sociale et artistique de Neuchâtel. Au-delà du DVD, la filmographie constitue un outil très appréciable pour qui s'intéresse à l'histoire locale et à l'histoire des représentations. Il faut rappeler qu'à son lancement en 2004, cette initiative constituait de par son recensement à l'échelle cantonale un "projet pionnier ». D'autres tomes semblent devoir suivre, peut-être sous une autre forme, en adéquation avec les «mutations profondes qui affectent les moyens de communiquer les archives» (p.19) en termes de métadonnées et d'accessibilité dématérialisée aux documents. Si une partie du corpus est déjà consultable en ligne, comme le CinéJournal suisse via la Mémobase de Mémoriav par exemple (http://memobase.ch/), facilitant ainsi la consultation des films à distance, il semble souhaitable, en effet, que les précieuses informations compilées dans cette filmographie soient, à terme, plus largement accessibles elles aussi. Notons toutefois qu'à ce jour, les notices jusqu'aux années 1940 sont disponibles en ligne via le portail des archives neuchâteloises (www.archivesne.ch). Cependant, on suivra avec intérêt la manière dont sera mise en œuvre le prochain tome, permettant d'observer la profonde transformation de la pratique filmographique que laisse envisager la forme numérique. 